\title{
Extended Floating Car Data System - Experimental Study -
}

\author{
R. Quintero, A. Llamazares, D. F. Llorca, M. A. Sotelo, L. E. Bellot, O. Marcos, I. G. Daza, C. Fernández
}

\begin{abstract}
This paper presents the results of a set of extensive experiments carried out in daytime and nighttime conditions in real traffic using an enhanced or extended Floating Car Data system (xFCD) that includes a stereo vision sensor for detecting the local traffic ahead. The detection component implies the use of previously monocular approaches developed by our group in combination with new stereo vision algorithms that add robustness to the detection and increase the accuracy of the measurements corresponding to relative distance and speed. Besides the stereo pair of cameras, the vehicle is equipped with a low-cost GPS and an electronic device for CAN Bus interfacing. The xFCD system has been tested in a 198-minutes sequence recorded in real traffic scenarios with different weather and illumination conditions, which represents the main contribution of this paper. The results are promising and demonstrate that the system is ready for being used as a source of traffic state information.
\end{abstract}

\section{INTRODUCTION}

The so-called Floating Car Data (FCD) refers to technology that collects traffic state information from a set of individual vehicles which float in the current traffic. Each vehicle, which can be seen as a moving sensor that operates in a distributed network, is equipped with global positioning and communication systems, transmitting its global location, speed, and direction to a central control unit that integrates the information provided by each one of the vehicles. FCD systems are being increasingly used in a variety of important applications since they overcome the limitations of fixed traffic monitoring technologies [1]. If this system achieves a sufficient penetration rate (1.5\% as described in [2]), the service quality in urban traffic would be sufficient. The most representative FCD projects in Japan, Europe and the United States until 2005 are described in [3].

The basic data provided by FCD systems can be extended (xFCD) using new specific devices and sensors endowed in modern vehicles [4], [5]. Such data can be exploited to extend the information horizon including traffic, weather, road management, and safety applications [3]. A second generation of xFCD has been recently proposed by including vision-based sensors in order to estimate the local traffic conditions. For example in [6] a stereo vision-based detection module is used as a vehicle ahead checker (excluding lateral road lanes) in order to validate the traffic alarms generated by the traffic level analyser.

R. Quintero, D. F. Llorca, M. A. Sotelo, L. E. Bellot, O. Marcos, and C. Fernández are with the Computer Engineering Department, Polytechnic School, University of Alcalá, Madrid, Spain. email : raul. quintero, llorca, sotelodaut.uah.es

A. Llamazares and I. G. Daza are with the Department of Electronics, Polytechnic School, University of Alcalá, Madrid, Spain. email: angel. llamazares@depeca.uah.es
Based on our previous works related with vision-based vehicle detection in the context of intelligent vehicles applications [7], [8], [9], we have proposed to use the information provided by the cameras to supply $\mathrm{xFCD}$ systems with a more representative measurements of the traffic conditions [10], [11]. A set of monocular vision-based modules (forward-rear and side-looking) were used to have a nearly 360 degree field of view. Instead of excluding lateral lanes [6] the relative distance and speeds of the vehicles detected in adjacent lanes were incorporated to the $\mathrm{xFCD}$ structure providing variables such as the local traffic load (number of vehicles, road capacity, etc.) and the average road speed (not only the floating vehicle speed) of the specific local area. Thus, a more detailed description (less discrete) of the traffic state can be obtained (for example, in cases where the floating vehicle is stopped in a congested lane but adjacent lanes have different levels of congestion).

In this paper we describe an improved approach of the vision-based vehicle detection systems described in [10] and [11], by means of the use of stereo vision. Stereo information improves both the detection performance and the accuracy of the measurements (host-to-vehicle $-\mathrm{H} 2 \mathrm{~V}$ - relative distance and speed). Previous results [11] were obtained in sequences of a few seconds (800 frames). Here we provide extensive results in sequences of a total duration of 198 minutes (428400 frames, $36 \mathrm{fps}$ ) recorded in real traffic scenarios with different weather (rainy/cloudy) and lighting conditions (nighttime/daytime), and different levels of congestion.

The remainder of this paper is organised as follows: Section II provides a global overview of the system. The description of the stereo vision-based vehicle detection system is presented in Section III. The experimental results are listed in Section IV, including sensor accuracy analysis. Finally, conclusions and future works are discussed in Section V.

\section{SYSTEM OVERVIEW}

The experimental vehicle used in this work is a car-like robot (a modified Citröen C4) which can be seen in Figure 1. It has an onboard computer housing the image processing system, a RTK-DGPS which is connected via RS232 serial port, a pair of synchronized low cost digital cameras connected via FireWire port, a specific electronic device for CAN Bus interfacing and a cellular communication system. The stereo vision sensor uses $320 \times 240$ pixel greyscale images with a baseline of approximately $300 \mathrm{~mm}$ and a focal length of $8.5 \mathrm{~mm}$.

The global architecture of the system can be seen in Figure 2. The results obtained by the stereo vision module are combined with the GPS measurements and the data provided 


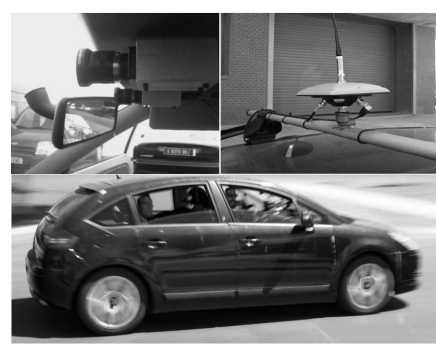

Fig. 1. Top left: Low cost stereo vision sensor. Top right: RTK-DGPS. Bottom: Experimental vehicle (modified Citröen C4).

by the CAN bus in order to have globally referenced traffic state information. Among the variables that are directly (or indirectly, after some processing) available via CAN Bus we remark: speed, acceleration, number of stop\&go (based on the speed), outside temperature and humidity, windshield wiper state, different lights state (fog, emergency, high beams, indicators, etc.), number of gear changes, number of lane changes (based on the indicator lights), etc.

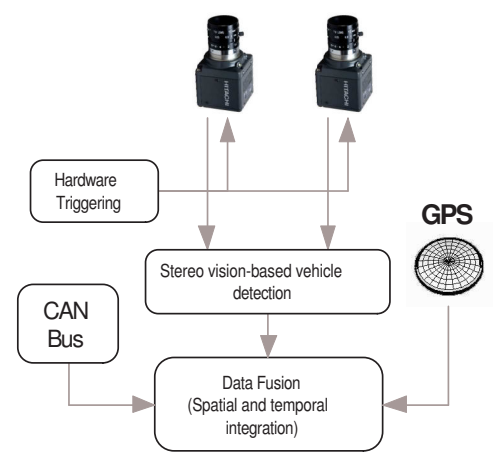

Fig. 2. Global architecture of the xFCD system

\section{VISION-BASED VEHICLE DETECTION}

The global scheme of the proposed vision-based vehicle detection system is depicted in Figure 3. The first step consists of reducing the searching space in the image plane by detecting the road lane markings. The detected lanes are used as the guidelines that drive the vehicle searching process. Lane markings are detected using gradient information in combination with a local thresholding method which is adapted to the width of the projected lane markings. Then, clothoid curves are fitted to the detected markings [11]. Then several region of interests (ROIs) generation modules are triggered in parallel.

Monocular ROIs are selected by combining white tophat and canny features with different types of symmetries (grey level, vertical edges and horizontal edges symmetries) as described in [11]. Stereo processing results in a dense disparity map which allows the 3D position and the relative speed of the vehicles ahead to be estimated accurately. The camera pitch angle is estimated dynamically by means of the so-called virtual disparity map from which regions corresponding to the ground-plane can easily be removed

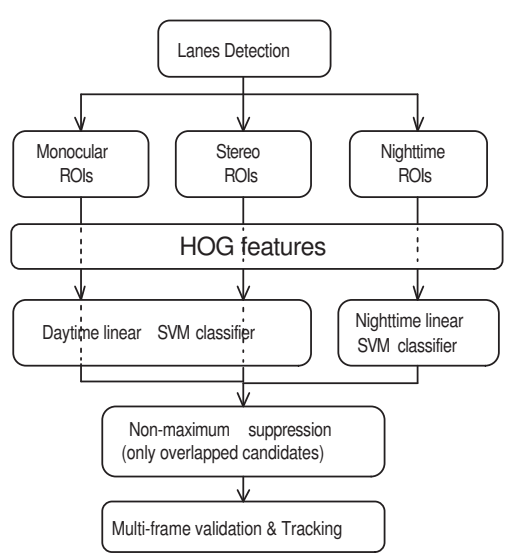

Fig. 3. Global overview of the vision-based vehicle detection algorithm.

[12]. One thus obtains a more precise specification of the areas of the ground where vehicles are to be expected (see Figure 4). Stereo ROIs are then computed by counting the number of depth features corresponding to the filtered dense disparity map of locations selected by means of perspective constraints (flat-world assumption) and prior knowledge of target objects (with tolerances). In particular, the locations where the number of depth features exceeds a certain fraction of the window area are passed on to subsequent modules, thus ensuring that each candidate region corresponds to a real 3D object.

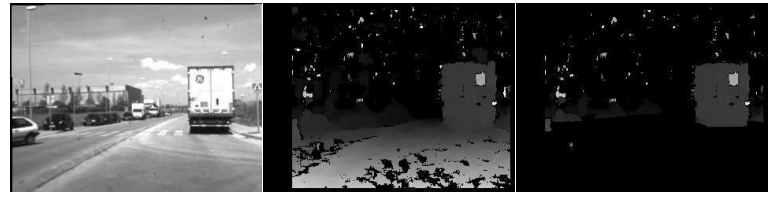

Fig. 4. From left to right: original image, dense disparity map, and filtered map (without ground-plane points or points that are very high).

Nighttime ROIs selection mechanism is firstly based on adaptive thresholding. Candidates are then obtained by searching pair of clusters (which are usually rear lights) with similar vertical location, using again the flat-world assumption, perspective constraints and prior knowledge of target objects. Although this is a simple method, it is very effective in practice. It provides a low number of false positives since the adaptive thresholding mostly highlights the rear lights of the vehicles (see Figure 5).

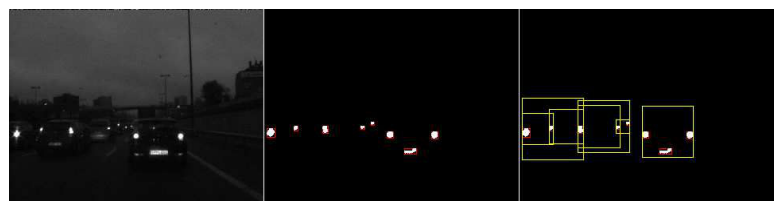

Fig. 5. From left to right: original image, individual clusters after adaptive thresholding and selected ROIs.

The three ROIs selection modules provide different candidates, some of them overlapped as they can refer to the 
same vehicle. All these candidates are classified by means of linear SVM classifiers [13] in combination with histograms of oriented gradients (HOG) features [14]. Two specialized classifiers have been trained depending on the lighting conditions. Candidates selected by monocular and stereo modules are classified using a daytime SVM classifier. On the other hand, a nighttime SVM classifier is used for nighttime ROIs (see Figure 3). Figure 6 shows some of the positive and negative training samples. The number of samples used for developing the training test differs depending on the classifier. Daytime classifier was trained with 19031 negative and 9248 positive samples. Nighttime classifier was obtained with 2486 negative and 1847 positive samples. The number of samples used in nighttime scenarios is lower because the intra-class variability is much lower than in daytime conditions.

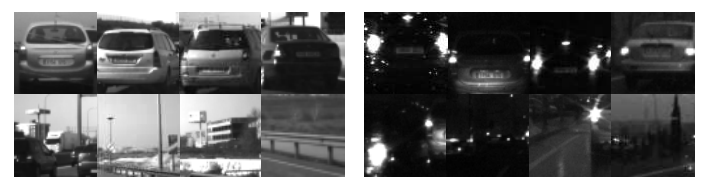

Fig. 6. Upper row: positive samples. Lower row: negative samples. Left: daytime samples. Right: nighttime samples.

The three detection modules may generate candidates related with the same vehicle. Accordingly a kind of nonmaximum suppression technique is used to group multiple overlapped candidates, trying to generate only one candidate per vehicle. In practice, we have observed that good candidates (candidates that are well fitted to the actual vehicle contour) usually provide SVM results farther to the hyperplane that separates the vehicle and non-vehicle classes, i.e., their classification result has higher confidence. Thus, we use the distance to the hyperplane as the main variable to select the best candidate of a set of overlapped candidates (overlapping has to be larger than $70 \%$ of the area of the smaller candidate).

Finally, the vehicles detected in this single-frame way are passed to the multi-frame validation and tracking module (see Figure 3). A predefined number (empirically set to 3) of consecutive identifications of an object classified as a vehicle triggers the data association and tracking stages. The data association problem is addressed by using feature matching techniques. Harris features are detected and matched between two consecutive frames, as in [11]. Tracking is implemented using a Kalman filter with a constant velocity model [11].

\section{EXPERIMENTAL RESULTS}

\section{A. System validation}

In a first experiment, we evaluated the different parameters provided by the vision sensor, i.e., $\mathrm{H} 2 \mathrm{~V}$ relative distance and speed, together with their corresponding errors. A set of sequences was recorded in which a driver was requested to perform an overtaking manoeuvre in a two-lane road at approximately $25 \mathrm{~km} / \mathrm{h}$. A vehicle was parked in the righthand (non-overtaking) lane, so that the host vehicle overtakes it at a relative speed of around $25 \mathrm{~km} / \mathrm{h}$. Besides the stereo vision sensor, two DGPSs are used, one placed at the lead vehicle's position and the other on board the host vehicle (see Figure 7). The measurements supplied by these DGPSs (after linear interpolation due to their low sampling frequency $5 \mathrm{~Hz}$ ) are taken to be the ground truth.

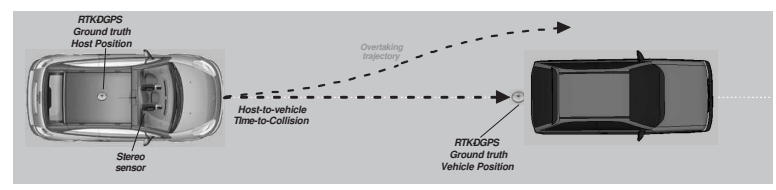

Fig. 7. Scheme of the manual driving experiment.

Figure 8 plots the stereo and the DGPS distance estimates, including the absolute error and the stereo depth estimation uncertainties [15]. The DGPS H2V, which is taken to be the ground truth, lies mostly within the limits of the stereo depth estimate including their corresponding uncertainties. As can be observed, the greater the depth the greater the absolute error. Depth estimates may not be reliable at long distances (absolute errors). However, the absolute error decreases as the $\mathrm{H} 2 \mathrm{~V}$ distance decreases.

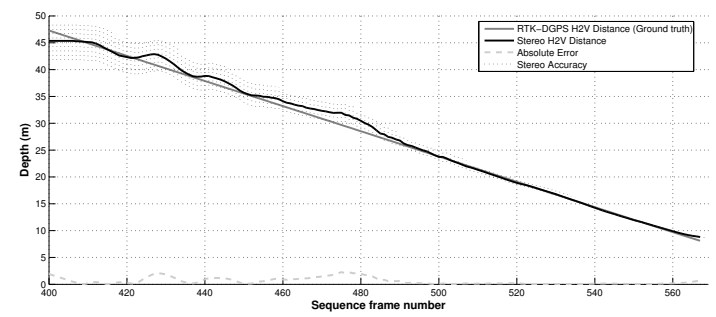

Fig. 8. Stereo and DGPS H2V relative distance including absolute and depth estimation errors in the experiment in which the car is overtaken.

Although the depth estimation errors are sufficiently small for the precision required in the present application, those of the discrete estimate of the relative speed from the Kalman state variable are not. As demonstrated in [16] discrete difference between two noisy depth estimation values introduces a considerable error in the relative speed computation for noninfinitesimal $\Delta t$, and this clearly limits the vision system's precision. Those authors propose an optimal value for $\Delta t$ which is directly proportional to depth. In the present case, we define a practical value of $\Delta t=1 \mathrm{seg}$ and compute the average speed of the last 25 frames. This approach is very effective in practice. Figure 9 depicts the relative speed obtained from the CAN bus (recall that the leading vehicle is parked in this experiment), that computed by means of DGPS $\mathrm{H} 2 \mathrm{~V}$ distance values, the discrete relative speed provided by the Kalman filter, and the relative speed computed at each second. One observes that the discrete values of the stereo relative speed are not at all useful. However, the proposed approach described above provides relative speed estimates that are accurate enough for the application's requirements the root-mean-squared-error (RMSE) is around $3 \mathrm{~km} / \mathrm{h}$. 


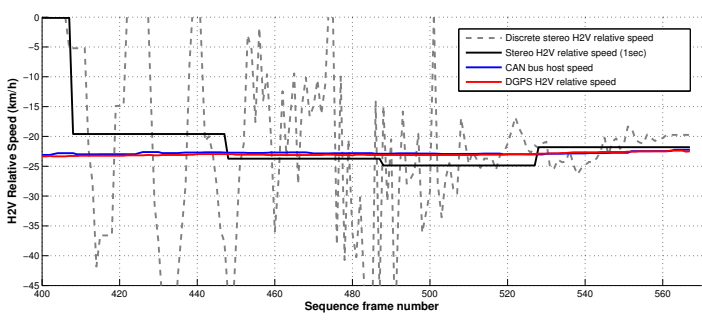

Fig. 9. H2V relative speed $(\mathrm{km} / \mathrm{h})$ taken from the CAN bus (the leading vehicle is parked), and from the DGPS depth values and the discrete stereo measurements, and estimated by averaging the discrete stereo values at each second, in the experiment where the car is overtaken.

\section{B. SVM Classifier}

As described in Section III, the candidates are classified by means of two linear SVM classifiers (daytime and nighttime). In order to define the SVM decision thresholds we use the ROC curves defining the work points in terms of the relationship between the Detection Rate (DR) and the False Positive Rate (FPR). In these experiments $2 / 3$ of the samples were used for training and $1 / 3$ for test. The ROC curves are showed in Figure 10. On the one hand, the decision threshold for the daytime classifier is fixed to 0.03 with a DR of $96.1 \%$ and a FPR of $6.4 \%$. On the other hand the decision threshold for the nighttime classifier is fixed to 0.07 with a DR of $91.4 \%$ and FPR of $7.8 \%$. We have to consider that these results are single-frame results, so they will be improved in multi-frame validation and tracking stages. The final classifier is trained with all the available samples.

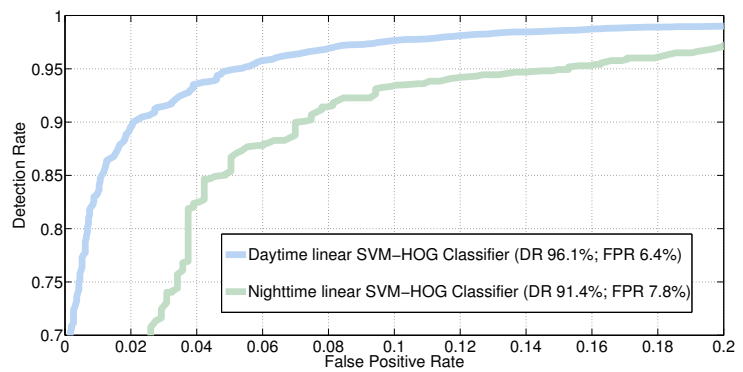

Fig. 10. ROC curves for both daytime and nighttime linear SVM classifiers.

\section{C. $x F C D$ results}

The proposed system was tested on data from real traffic conditions. A set of video sequences were recorded on the same route through the Madrid (Spain) M-30 highway (see Figure 11). The route distance is approximately 15 kilometres and it was driven up to 4 times, going and returning from the A and B points highlighted in Figure 11. Accordingly, a total of 150 kilometres were driven approximately. The video sequences were recorded during the same day, from 7:20 AM to 10:38 AM, including different lighting (nighttime/daytime) and weather (rainy/cloudy) conditions, as well as different levels of congestion.

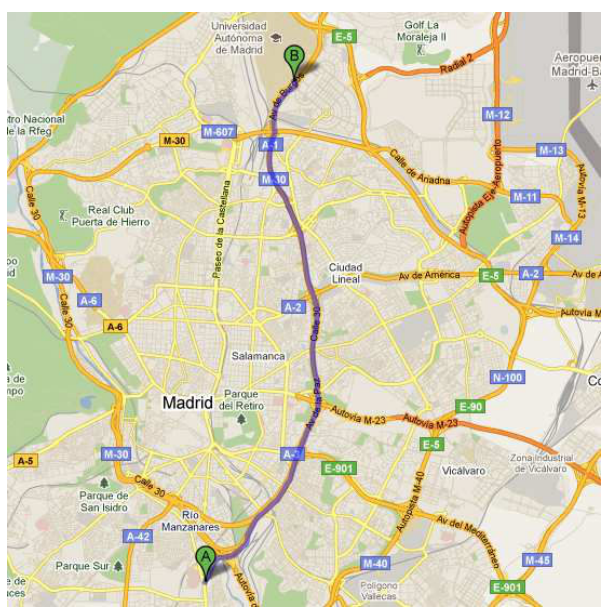

Fig. 11. Route through the Madrid (Spain) M-30 highway followed during the experiments.

Global results are presented in Table I. We have labelled the total amount of vehicles in range in all the sequences (ground truth). Note that we distinguish between true positives and vehicles that are detected from a previously detected vehicle (identifier exchanged). In both cases we consider the vehicle as detected, assuming that some errors will appear in the relative distance computation when a new vehicle is associated with a previously tracked one. Accordingly, we obtain a detection rate of $88.44 \%$ and $90.99 \%$ in nighttime and daytime conditions respectively. 0.84 false positives per minute are obtained in nighttime conditions whereas 0.69 false positives per minute are detected in daytime conditions. In addition, the $6.67 \%$ and the $5.82 \%$ of the vehicles are counted more than one time in nighttime and daytime conditions respectively (i.e. a new identifier is generated).

Figures 12 and 13 depict some of the variables provided by the system in two sequences (daytime and nighttime respectively) with different levels of traffic congestion. The following variables are drawn: host speed (via CAN bus interface), the average road speed, the distance between the host vehicle and the vehicle ahead, and the number of vehicles detected per each frame. As can be observed, there is a strong relationship between the road speed and the $\mathrm{H} 2 \mathrm{~V}$ distance. In fact we consider this variable as one of the most relevant in terms of traffic congestion. The maximum number of vehicles detected per frame is 6 (note that the minimum number is 1 since we always count the host vehicle). The number of vehicles detected does not seem to affect to the average road speed computation which implies that the relative distances of the vehicles detected in adjacent lanes and the proposed method for computing the relative speed are coherent up to a point (extensive ground truth data will be needed to assure the actual goodness of the relative distance and speed measures).

The average difference between the host speed and the average road speed is around $0.4 \mathrm{~km} / \mathrm{h}$. This can be explained by the fact that the host vehicle is driving in the middle 
TABLE I

GLOBAL XFCD RESULTS

\begin{tabular}{|c|c|c|c|c|c|c|c|}
\hline & \# frames & $\begin{array}{c}\text { \# vehicles in range } \\
\text { (ground truth) }\end{array}$ & $\begin{array}{c}\text { True } \\
\text { Positives }\end{array}$ & $\begin{array}{c}\text { Identifier } \\
\text { exchanged }\end{array}$ & $\begin{array}{c}\text { Missed } \\
\text { vehicles }\end{array}$ & $\begin{array}{c}\text { False } \\
\text { Positives }\end{array}$ & $\begin{array}{c}\text { Multiple } \\
\text { counts }\end{array}$ \\
\hline Nighttime (from 7:20am to 8:35am) & 161907 & 727 & 625 & 18 & 84 & 63 & 50 \\
\hline Daytime (from 8:35am to 10:38am) & 266493 & 1133 & 1007 & 24 & 102 & 86 & 66 \\
\hline Total (198 minutes) & 428400 & 1860 & 1632 & 42 & 186 & 149 & 116 \\
\hline
\end{tabular}

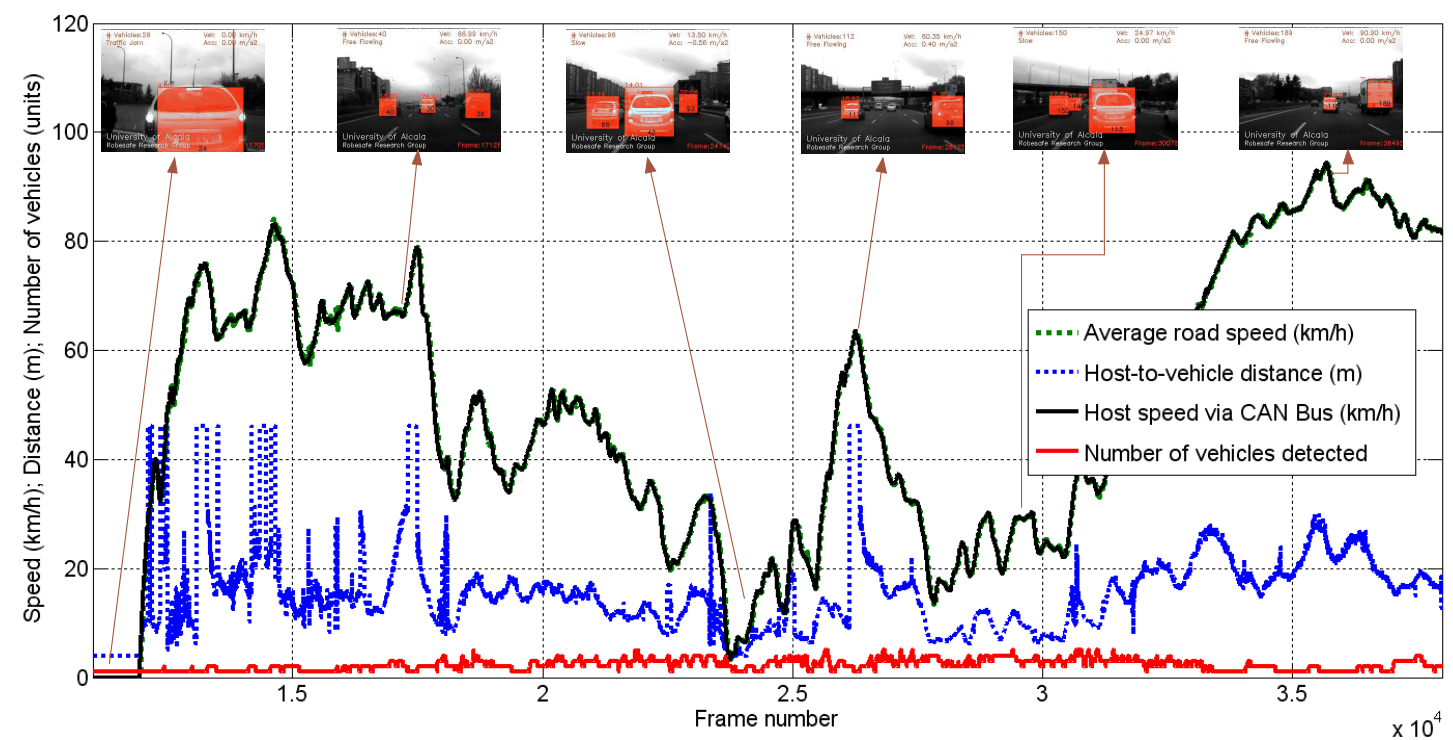

Fig. 12. Results in daytime, cloudy and rainy conditions with different levels of traffic congestion.

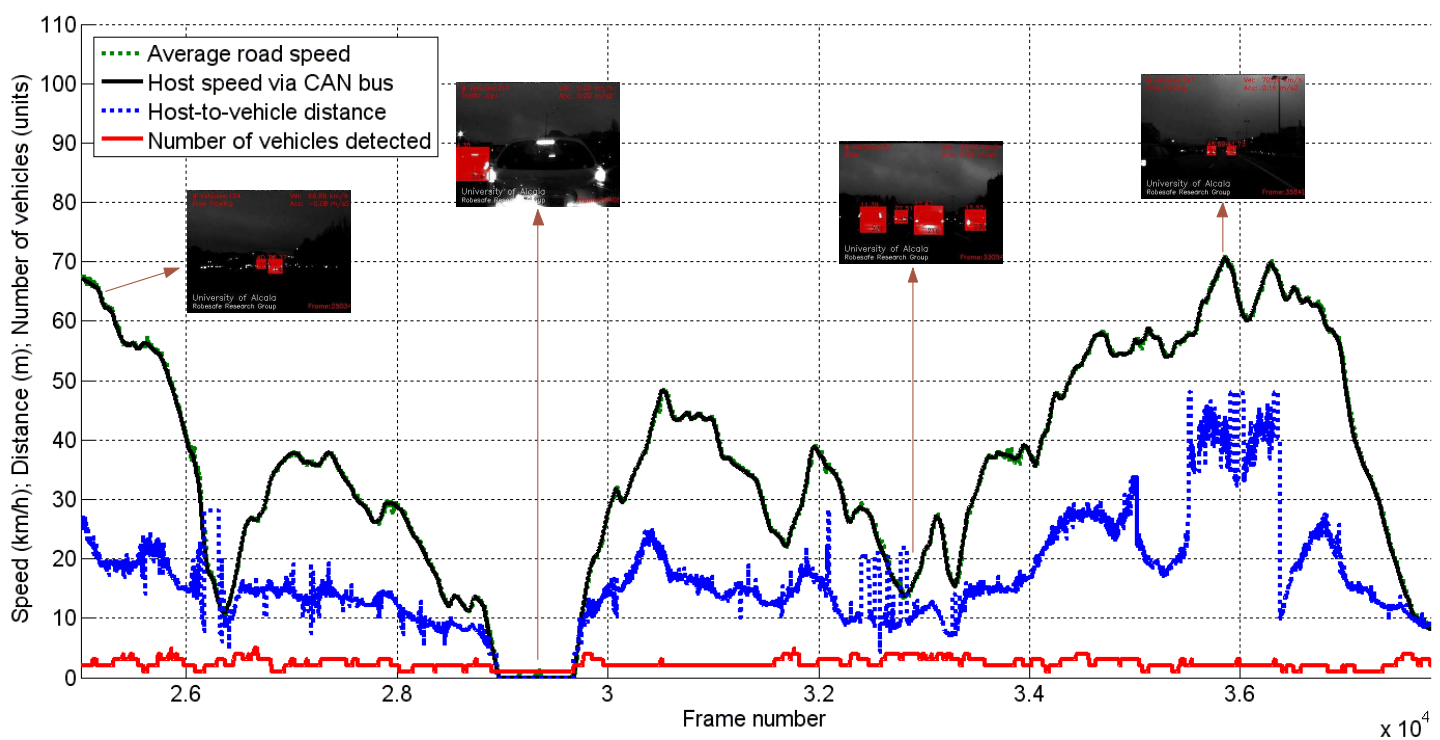

Fig. 13. Results in nighttime, cloudy and rainy conditions with different levels of traffic congestion. 
lane of a three lane highway most of the time, so the higher speed of the vehicles located on the left lane is compensated somehow with the lower speed of vehicles located on the right lane. However we have detected some cases where the host vehicle is stopped (host speed equal to $0 \mathrm{~km} / \mathrm{h}$ ) whereas the vehicles located on the left lane are moving at speeds up to $10 \mathrm{~km} / \mathrm{h}$. This effect can be observed in Figure 14. In some cases the difference between the host speed and the average road speed achieves more than $3 \mathrm{~km} / \mathrm{h}$.

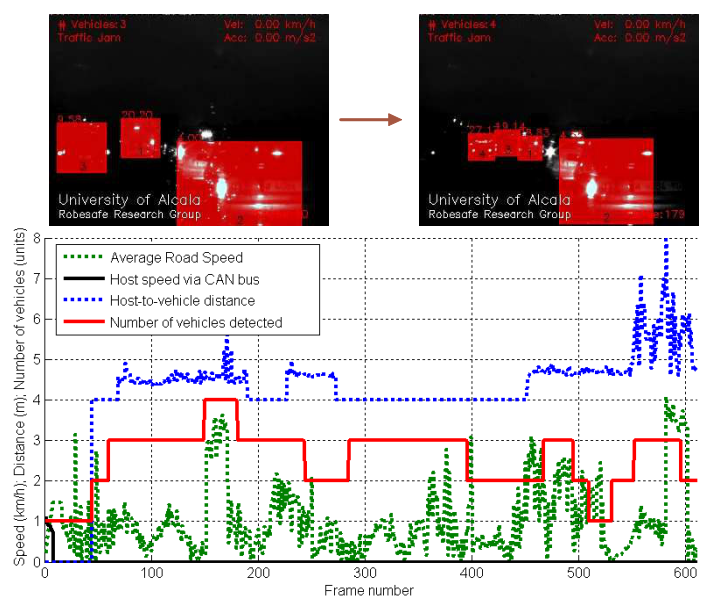

Fig. 14. Specific situation: host vehicle is stopped whereas the vehicles located on the left lane are moving.

\section{CONCLUSIONS AND FUTURE WORKS}

This paper presented the results of an xFCD system equipped with a stereo vision-based local traffic detector in a set of extensive experiments (198 minutes) carried out in real traffic conditions with different lighting and weather conditions as well as different levels of traffic congestion. The vision-based vehicle detection system combines different approaches (monocular, stereo and nighttime) to supply generic obstacles that are classified by means of two linear SVM classifiers (daytime/nighttime) and HOG features. The vehicles are then validated in a multi-frame fashion and tracked using a Kalman filter. The proposed approach provides data, not only from the host vehicle, but from the vehicles located in the field of view of the host, including the vehicle ahead and the vehicles located in adjacent lanes (when available). Thus, standard variables such as the host vehicle speed (via CAN bus) can be enriched and supported with new variables such as the distance to the vehicle ahead, the average road speed, the number of vehicles in range, etc. Although the use of stereo vision implies to manage some errors when estimating the relative distance (errors that are squared proportional to the depth), it allows us to have an accurate estimation of the relative speed by integrating relative distance values during 1 second. The global results show that the proposed approach produces good results in different traffic conditions, succeeding when detecting the traffic congestion.
Although the results are promising, new advances have to be developed to improve the detection rate and reduce the number of false positives. A more sophisticated ensemble classifier will be introduced using different classifiers depending on the lane, and including a new class for trucks since most of the missed vehicles are trucks.

Finally, a set of experiments have to be addressed to clearly define the limits of the proposed system. We will compare the $\mathrm{xFCD}$ results with data provided by fixed loops that are installed throughout the same highway used in the experiments. New vehicles (fleet) will be used, and xFCD will be combined with data from fixed loops to assess applications in the context of traffic management and control.

\section{ACKNOWLEDGMENTS}

This work has been supported by the Spanish Ministry of Development by means of Research Grant GUIADE P9/08.

\section{REFERENCES}

[1] J. E. Naranjo, F. Jiménez, F. J. Serradilla and J. G. Zato. ”Comparison between Floating Car Data and Infrastructure Sensors for Traffic Speed Estimation". in Proc. of IEEE ITSC, Madeira, 2010.

[2] C. Demir, et al., "FCD for Urban Areas: Method and Analysis of Practical Realizations," in Proc. of the 10th Intelligent Transportation Systems World Congress, Madrid, Spain, 2003.

[3] R. Bishop, Intelligent Vehicle Technologies and Trends, Artech House, Boston, Mass, USA, 2005.

[4] W. Huber, M. Lädke and R. Ogger. "Extended floating-car data for the acquisition of traffic information". in Proceedings of the 6th World Congress on Intelligent Transport Systems, 1999.

[5] A. Escher. "Enhanced floating car data approaches enabled by GST". in Proc. of 12th World Congress on Int. Transp. Systems. 2005.

[6] S. Messelodi, C. M. Modena, M. Zanin et al., "Intelligent extended floating car data collection," Expert Systems with Applications, vol. 36, no. 3, part 1, pp. 4213-4227, 2009.

[7] S. Álvarez, M. A. Sotelo, M. Ocaña, D. F. Llorca, I. Parra and L. M. Bergasa. "Perception advances in outdoor vehicle detection for automatic cruise control". Robotica, vol 28, no. 5, pp. 765-779.

[8] M. A. Sotelo and J. Barriga, "Blind spot detection using vision for automotive applications," Journal of Zhejiang University: Science A, vol. 9, no. 10, pp. 1369-1372, 2008.

[9] D. Balcones, D. F. Llorca, M. A. Sotelo, et al., "Realtime vision-based vehicle detection for rear-end collision mitigation systems," in Proc. of the 12th International Conference on Computer Aided Systems Theory (EUROCAST '09), vol. 5717 of LNCS, pp. 320-325, 2009.

[10] D. F. Llorca, S. Sánchez, M. Ocaña, M. A. Sotelo. "Vision-Based Traffic Data Collection Sensor for Automotive Applications". Sensors, 2010, vol 10, pp. 860-875.

[11] D. F. Llorca, M. A. Sotelo, S. Sánchez, M. Ocaña, J. M. RodríguezAscariz, M. A. García-Garrido. "Traffic Data Collection for Floating Car Data Enhancement in V2I Networks", EURASIP Journal on Advances in Signal Processing, vol. 2010, art. ID 719294, 13 pages.

[12] D. F. Llorca, M. A. Sotelo, I. Parra, J. E. Naranjo, M. Gavilán, and S. Álvarez, "An experimental study on pitch compensation in pedestrian-protection systems for collision avoidance and mitigation," IEEE Trans. on Int. Transp. Systems, vol. 10, no. 3, pp. 469-474, 2009.

[13] C. J. Christopher Burges, "A tutorial on support vector machines for pattern recognition," Data Mining and Knowledge Discovery, vol. 2, no. 2, pp. 121-167, 1998.

[14] N. Dalal and B. Triggs, "Histograms of oriented gradients for human detection," in Proc. of the IEEE Computer Society Conference on Computer Vision and Pattern Recognition, pp. 886-893, 2005.

[15] D. F. Llorca, M. A. Sotelo, I. Parra, M. Ocaña, and L. M. Bergasa, "Error analysis in a stereo vision-based pedestrian detection sensor for collision avoidance applications", Sensors, vol. 10, no. 4, pp. 3741$3758,2010$.

[16] G. P. Stein, O. Mano, and A. Shashua, "Vision-based ACC with a single camera: bounds on range and range rate accuracy," in Proc. IEEE Intelligent Vehicles Symposium., 2003, pp. 120-125. 